\title{
Structural Design of Nozzle Based on Hybrid Laser/Water-jet Scribing Technology
}

\author{
Yuhong Long ${ }^{\mathrm{a}}$, Tanggao Feng ${ }^{\mathrm{b}}$, Jiading $\mathrm{Bao}^{\mathrm{c}}$, and Youqun Tong ${ }^{\mathrm{d}}$
}

School of Mechanical \& Electrical Engineering, Guilin University of Electrical Technology, Guilin

$$
\text { 541004, China; }
$$

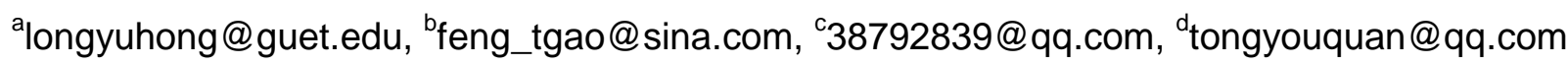

Keywords: scribing, conical nozzle, fluid field simulation.

\begin{abstract}
In order to research the effect of nozzle geometric parameters on the jet flow performance in processing a thin and sensitive material. A design of two nozzles with different structure has been proposed for hybrid laser/water-jet scribing technology. In this paper, computational fluid dynamics (CFD) method was used to simulate and analyze the gas-water two phase flows of water jets. Compared with cylindrical nozzle, the results show that conical nozzle obtains a greater pressure field and more stabilized velocity field. As a result, by comparing the optimization can get the optimum nozzle structure parameters.
\end{abstract}

\section{Introduction}

In recent years, hybrid laser/water-jet scribing technology is a frontier research topic. Given that the quality problem of the thin, sensitive and brittle material scribing, a novel laser and water-jet scribing process has been put forward to improve processing quality and meet request of precision. Conventionally, laser processing like $\mathrm{CO}_{2}$ and $\mathrm{Nd}$ :YAG lasers are used in a variety of material processing applications for a wide range of materials, and there appears to be no limit to the range of possible materials, which include metals, ceramics, composite materials, polymers, semiconductors, and biological tissue [1]. the laser beam is emitted by the use of high power laser irradiation on the surface of work-piece, local softening and melting work-piece by irradiation area so as to achieve the purpose of the cut, and then water jet will wash molten slag molten slag from work-piece surface, which is soften by high energy of laser. The nozzle is the most important terminal actuators as the equipment of water jet in process, its structural parameters will eventually decide to water jet equipment property. However, it is difficult to provide a suitable nozzle's structure parameter. To obtain its structure parameter, numerical simulation has been carried out in FLUENT software.

Using experimental research method, it need process a large number of nozzles of different structure. It is difficult to evaluate flow field characteristics, and cost is expensive. While numerical simulation technology can avoid these problems well [2]

\section{Numerical Algorithm}

\subsection{The Control Equations.}

CFD technology is to solve the control equations (include momentum conservation equation, energy conservation equation, continuity equation and equation, etc.) of fluid mechanics by numerical calculation method[3].

Continuity equation is

$\frac{\partial_{\rho}}{\partial t}+\frac{\partial}{\partial x_{i}}\left(\rho u_{i}\right)=0$

Momentum conservation equation is

$$
\frac{\partial\left(\rho \mu_{i} \mu_{j}\right)}{\partial x_{i}}=\frac{-\partial p}{\partial x_{i}}+\frac{\partial}{\partial x_{j}}\left[\mu\left(\frac{\partial \mu_{i}}{\partial x_{j}}+\frac{\partial \mu_{j}}{\partial x_{i}}\right)\right]-\rho \overline{\mu_{i}^{\prime} \mu_{j}^{\prime}}
$$


Where $\mu$ is velocity of continuous phase, $\mathrm{i}, \kappa$ is the coordinate direction and the direction of the velocity components in the Coordinate system. The density of continuous phase and the pressure of continuous phase and molecular dynamic viscosity coefficient are $\rho, \mathrm{p}$ and $\mu$, respectively.

$\kappa$ equation is

$\frac{\rho \mu_{i} \partial_{k}}{\partial x_{j}}=\frac{\partial}{\partial x_{j}}\left[\left(\mu+\frac{\mu_{i}}{\sigma_{k}}\right) \frac{\partial k}{\partial x_{j}}\right]+\mu_{i} \frac{\partial \mu_{i}}{\partial x_{j}}\left(\frac{\partial \mu_{i}}{\partial x_{j}}+\frac{\partial \mu_{j}}{\partial x_{j}}\right)-\rho \varepsilon$

$\varepsilon$ equation is

$\frac{\rho \mu_{j} \partial_{\varepsilon}}{\partial x_{j}}=\frac{\partial}{\partial x_{j}}\left[\left(\mu+\frac{\mu_{t}}{\sigma_{\varepsilon}}\right) \frac{\partial \varepsilon}{\partial x_{j}}\right]+\frac{C_{1} \mu_{t} \varepsilon \partial \mu_{i}}{k \partial x_{j}}\left(\frac{\partial \mu_{i}}{\partial x_{j}}+\frac{\partial \mu_{j}}{\partial x_{i}}\right)-\rho C_{2} \frac{\varepsilon^{2}}{k}$

Where $\mu_{t}=\frac{C_{\mu} \rho \kappa^{2}}{\varepsilon}, \mu_{t}$ is turbulence kinetic viscosity coefficient. turbulent pulsating kinetic energy of unit mass of fluid and turbulent pulsating kinetic energy dissipation rate unit mass of fluid are respectively $\kappa$ and $\varepsilon$. Turbulent Prandtl number of $\kappa$ and $\varepsilon$ are $\sigma_{\kappa}$ and $\sigma_{\varepsilon}$, respectively.

\section{Numerical simulation}

\subsection{Geometric Model of Nozzle}

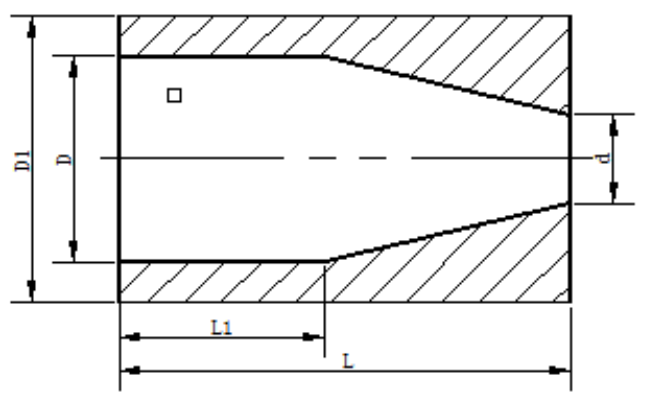

(a) conical nozzle

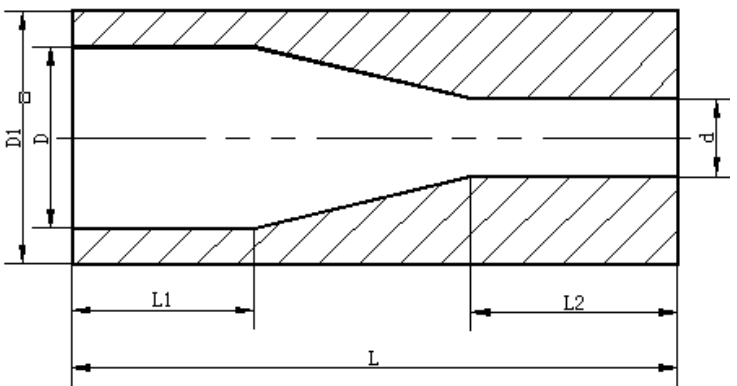

(b) cylindrical nozzle

Fig.1 section of nozzle

Table 1: two structure parameter comparing (unit: millimeter)

\begin{tabular}{|c|c|c|c|c|c|c|}
\hline Name & D1 & D & L1 & L2 & L & d \\
\hline Conical nozzle & 1.4 & 1 & 1 & 0 & 2.2 & 0.57 \\
\hline Cylindrical nozzle & 1.4 & 1 & 1 & 1.14 & 2.2 & 0.57 \\
\hline
\end{tabular}

\subsection{The division of the grid}

In GAMBIT, two nozzles (as showed in the Fig.1) have been divided into meshed structure. Due to the flow field of nozzle along outlet diameter direction is more intense than others. Local grid along diameter length direction is dense [4] .Based on the grid meshing theories; refined grid quality has been taken into consideration in this paper with using the structure of triangular grid. Triangular grid is affiliated with unstructured grid, which can eliminate the structural limits of the structure in the grid node. While node and element distribution obtain good controllability, boundary conditions has been handled. As showed in the Fig.2 below for the unstructured grid of cylindrical nozzle.

\subsection{Boundary conditions}

Fig.2: the unstructured grid of cylindrical nozzle

Computational domains of boundary conditions are set as shown in Fig. 3. Line AB is the velocity outlet of high pressure water, the velocity magnitude equal $12 \mathrm{~m} / \mathrm{s}$. Line BC,CD DE are wall boundary 
condition of nozzle. Line $\mathrm{AH}, \mathrm{GH}$ are the axis of symmetry and wall boundary condition of work-piece, respectively. Polygonal ABCDE is internal flow domain, and square EFGH is external flow domain (length $\times$ width $=5 \mathrm{~d} \times 20 \mathrm{~d}$, $\mathrm{d}$ is diameter of the nozzle exit)

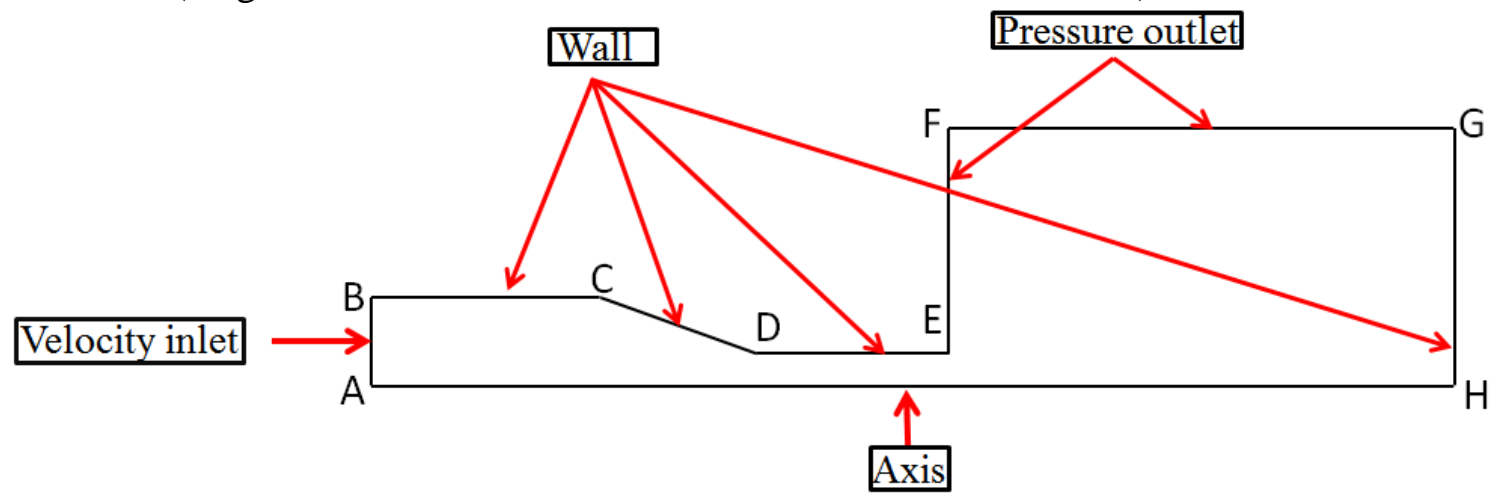

Fig. 3 boundary conditions sets

\section{Simulative Result and experimental verification}

We apply VOF model of mutiphase in FLUENT and use cooperatively standard $\kappa: \varepsilon$ model with standard wall functions [5]. The primary and secondary phases are defined: (a) Set the phase material of primary-phase (phase-1) to water-liquid and rename phase-1to water; (b) Similarly set the secondary-phase material to air. When everything is ready, running calculation will carry out iteration and calculation. As show in Fig. 4(a) and Fig. 4(b) are contours of total pressure of conical nozzle and cylindrical nozzle, respectively. Their velocity magnitudes along axial position are showed in Fig.5 (a) and Fig.5 (b).

In order to verify validity of simulative result, experimental facilities (beakers) are applied to measure velocity magnitude of nozzle exit. Experimental method is demonstrated as follow: when water-jet flow from nozzle inlet to nozzle outlet, the time (seconds) it takes under quantitative $1000 \mathrm{~mL}$ water-liquid circumstance [6].The average velocity expresses as follow:

$$
\mathrm{v}_{\mathrm{j}}^{\prime}=\frac{\omega}{\pi \mathrm{d}^{2} \mathrm{t}}
$$

Where $r_{0}=4 \times 10^{-5} \mathrm{~m}, \omega$, d are water-liquid volume and diameter of the nozzle exit ,respectively. Compared with theoretical velocity magnitude of expression 5 , simulative velocity magnitude in the fig.5 is reasonable.

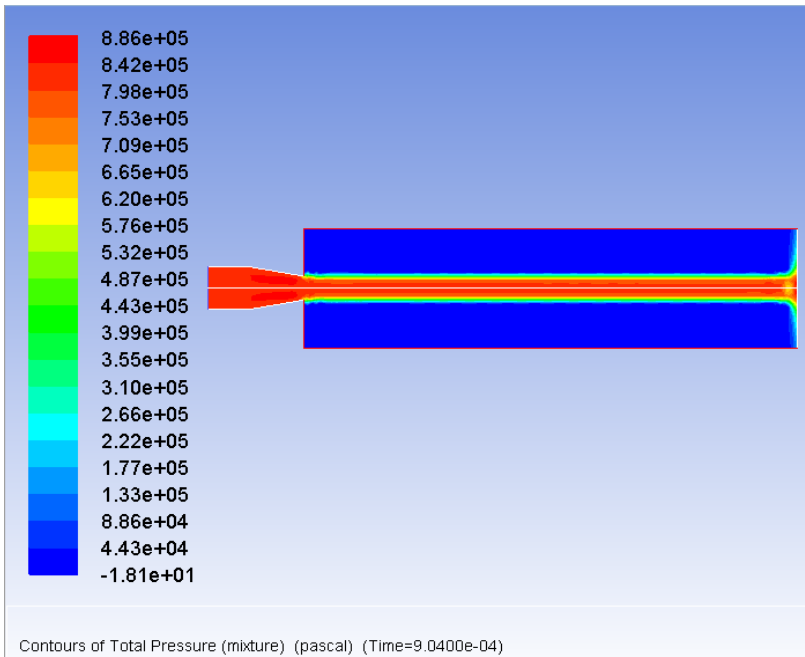

(a) conical nozzle contours of total pressure

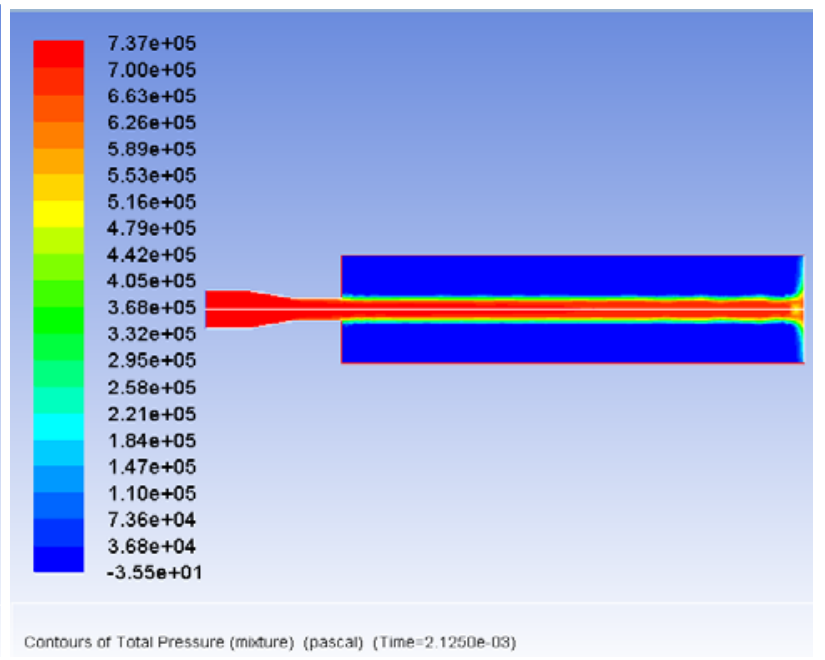

(b) cylindrical nozzle contours of total pressure

Fig.4 : contours of total pressure 


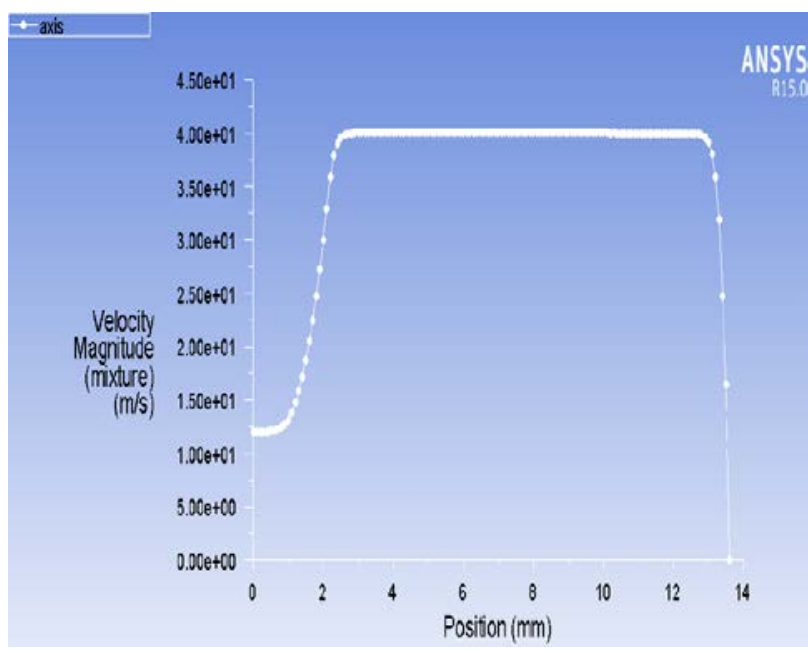

(a) conical nozzle

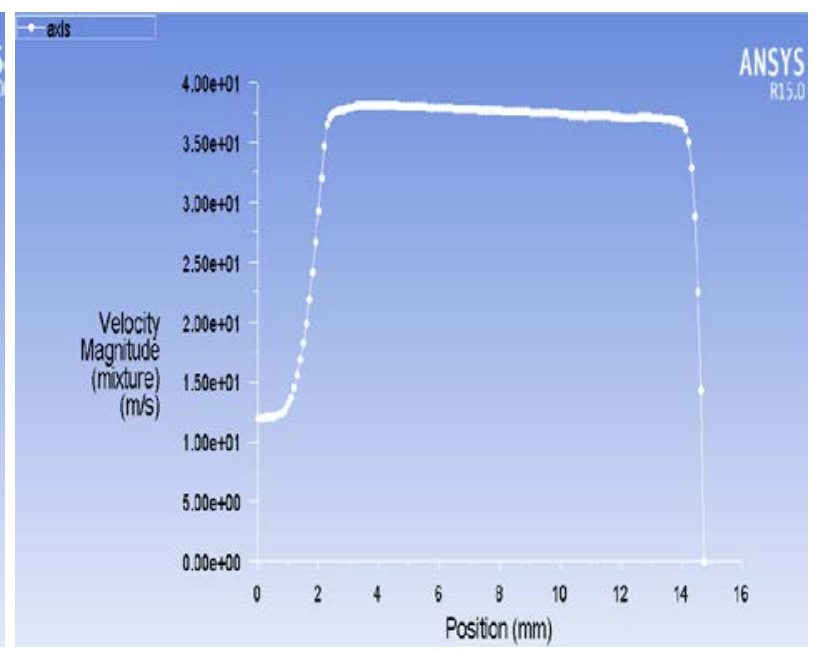

(b) cylindrical nozzle

Fig.5: xy plot of velocity magnitude

\section{Conclusions}

(1) The VOF model in FLUENT is used to analyze two phase flow of nozzle jet.While numerical simulation has has been conducted under the same simulative parameter condition, conical nozzle can provide a large pressure field and more stable velocity (approximate magnitude is $40 \mathrm{~m} / \mathrm{s}$ ) when axial position rang from $3 \mathrm{~mm}$ to $13 \mathrm{~mm}$. The result suggest that conical nozzle's performance is superior to cylindrical nozzle.

(2) When water-jet ejecting from nozzle outlet of $0.57 \mathrm{~mm}$ diameter get to the surface of work-piece, conical nozzle obtain a longer jet flow domain compared to cylindrical nozzle.

(3) Two sets of results show that there are a close ralationships between the distance of the workpiece and the length of water-jet. Only when water-jet's velocity is stable, work-piece will acquire maximum impact force.

\section{Acknowledgement}

This work was financially supported funded by National Natural Science Foundation of China (61366009), the key project of Guangxi Natural Science Foundation (2015GXNSFDA139036), the director Fund of Guangxi Manufacturing Systems and Advanced Manufacturing Technology Laboratory (13-051-09-004Z), and Guangxi Experiment Center of Information Science, Guilin University of Electronic Technology (20130313).

\section{References}

[1]. YangZhang.Structure Parameter Optimization of Water Jet Cutting Nozzle by Numerical Simulation.Machine Tool\&Hydraulics.Vol.42(2014)No.19,P.110-112.

[2]. Ng EY-K, Guannan D.The stability of 30- $\mu$ m-diameter water jet for jet-guided laser machining.The International Journal of Advanced Manufacturing Technology.Vol.78(2015)No. 11,p. 939-946.

[3]. Yong-hong Fu, Jia Cao, Fei Dong, et al. CFD Simulation of Laser Stable Water Beam in Micro Water-jet Guided Laser. Fluid \&Machinery. Vol.41(2013)No.8,p.21-26

[4]. Kalyanasundaram, D: Mechanics guided design of hybrid laser/waterjet system for machining of hard and brittle materials(PH.D, lowa state university,US,2009).p.25 
[5]. Towards Damage-free Mocro-fabrication of Silicon Substrates Using A Hybrid Laser-waterjet Technology(PH.D,the University of New South Wales, Australia,2012).p.61

[6]. Li-fang Liu, Chuan-zhen Huang, Hong-tao Zhu, et al.Simulation of Internal Flow Field of Non-Submerged Water Jet Nozzle Based on Fluent. Manufacturing Technology and Machine Tool.Vol.10(2010)No.5,p.56-59 